\title{
CORRECTIONS
}

\section{Author Correction: Progress in multiple sclerosis \\ — from diagnosis to therapy}

\section{Maria Trojano and Maria Pia Amato}

Nature Reviews Neurology (2018) https://doi.org/10.1038/nrneurol.2018.3

Published online 31 January 2018

In the originally published article, Maria Pia Amato's affiliation was incomplete - it should have included IRCCS Fondazione Don Carlo Gnocchi, Florence, Italy. This correction notice has been appended to the PDF version of the manuscript.

https://doi.org/10.1038/s41582-019-0179-z I Published online 1 April 2019 